\title{
ON SUBSEQUENTIAL CONVERGENCE OF BOUNDED SEQUENCES
}

\section{IBRAHIM ÇANAK AND ÜMITT TOTUR}

Received 28 September, 2014

\begin{abstract}
In this paper we establish some Tauberian-like conditions in terms of the weighted general control modulo of integer order to retrieve subsequential convergence of a sequence from its boundedness.
\end{abstract}

2010 Mathematics Subject Classification: 40E05

Keywords: subsequential convergence, weighted means, weighted general control modulo, slowly oscillating sequence

\section{INTRODUCTION}

Let $p=\left(p_{n}\right)$ be a sequence of nonnegative numbers with $p_{0}>0$ and

$$
P_{n}:=\sum_{k=0}^{n} p_{k} \rightarrow \infty \quad \text { as } n \rightarrow \infty .
$$

The weighted means of a sequence $\left(u_{n}\right)$ of real numbers are defined by

$$
\sigma_{n, p}^{(1)}(u):=\frac{1}{P_{n}} \sum_{k=0}^{n} p_{k} u_{k}
$$

for all nonnegative integers $n$.

We define the weighted mean method as follows:

Definition 1. If $\lim _{n \rightarrow \infty} \sigma_{n, p}^{(1)}(u)=s$, then we say that $\left(u_{n}\right)$ is said to be limitable to $s$ by the weighted mean method $\left(\bar{N}, p_{n}\right)$ and we write $u_{n} \rightarrow s\left(\bar{N}, p_{n}\right)$.

If $p_{n}=1$ for all $n$, then the corresponding weighted mean method is the $(C, 1)$ method of Cesàro.

The sequence $\Delta u=\left(\Delta u_{n}\right)$ of the backward differences of $\left(u_{n}\right)$ is defined by $\Delta u_{n}=u_{n}-u_{n-1}$, and $\Delta u_{0}=u_{0}$ for $n=0$.

The identity

$$
u_{n}-\sigma_{n, p}^{(1)}(u)=V_{n, p}^{(0)}(\Delta u),
$$


where $V_{n, p}^{(0)}(\Delta u)=\frac{1}{P_{n}} \sum_{k=1}^{n} P_{k-1} \Delta u_{k}$, is known as the weighted Kronecker identity.

We define $\sigma_{n, p}^{(m)}(u)$ and $V_{n, p}^{(m)}(\Delta u)$ by

$$
\sigma_{n, p}^{(m)}(u)= \begin{cases}\frac{1}{P_{n}} \sum_{k=0}^{n} p_{k} \sigma_{k, p}^{(m-1)}(u) & , m \geq 1 \\ u_{n} & , m=0\end{cases}
$$

and

$$
V_{n, p}^{(m)}(\Delta u)= \begin{cases}\frac{1}{P_{n}} \sum_{k=0}^{n} p_{k} V_{k, p}^{(m-1)}(\Delta u) & , m \geq 1 \\ V_{n, p}^{(0)}(\Delta u) & , m=0\end{cases}
$$

respectively (see [4]).

The weighted classical control modulo of $\left(u_{n}\right)$ is denoted by $\omega_{n, p}^{(0)}(u)=\frac{P_{n-1}}{p_{n}} \Delta u_{n}$ and the weighted general control modulo of integer order $m \geq 1$ of $\left(u_{n}\right)$ is defined by $\omega_{n, p}^{(m)}(u)=\omega_{n, p}^{(m-1)}(u)-\sigma_{n, p}^{(1)}\left(\omega^{m-1}(u)\right)$ (see [2]).

A new kind of convergence was defined by Dik [5] as follows.

Definition 2. A sequence $u=\left(u_{n}\right)$ is said to be subsequentially convergent if there exists a finite interval $I(u)$ such that all accumulation points of $\left(u_{n}\right)$ are in $I(u)$ and every point of $I(u)$ is an accumulation point of $\left(u_{n}\right)$.

It is clear from the definition that subsequential convergence implies boundedness. But the converse is not true in general. For example, $\left((-1)^{n}\right)$ is bounded, but it is not subsequentially convergent. The converse implication is true under some conditions imposed on the sequence.

The following theorem is a more general one stating that under which condition bounded sequences are subsequential convergent.

Theorem 1 ([5]). Let $\left(u_{n}\right)$ be a bounded sequence. If $\Delta u_{n}=o(1)$, then $\left(u_{n}\right)$ is subsequentially convergent.

Throughout this paper, we write $u_{n}=o(1)$ or $u_{n}=O(1)$ rather than $\lim _{n} u_{n}=0$ or $\left(u_{n}\right)$ is bounded for large enough $n$.

Example 1. Using Theorem 1, one can easily show that the sequence $\left(u_{n}\right)=$ $(\sin \sqrt{n})$ is subsequentially convergent. Indeed, the sequence $\left(u_{n}\right)$ is bounded. From the fact that

$$
\begin{aligned}
\left|\Delta u_{n}\right|=|\Delta \sin (\sqrt{n})| & =|\sin (\sqrt{n})-\sin (\sqrt{n-1})| \leq|\sqrt{n}-\sqrt{n-1}| \\
& =\left|\frac{1}{\sqrt{n}+\sqrt{n-1}}\right|=o(1),
\end{aligned}
$$


it follows that $\Delta u_{n}=o(1)$. Thus by Theorem 1 , the sequence $\left(u_{n}\right)$ is subsequentially convergent.

Definition 3 ([9]). A sequence $\left(u_{n}\right)$ is slowly oscillating if

$$
\lim _{\lambda \rightarrow 1+} \varlimsup_{n} \max _{n+1 \leq k \leq[\lambda n]}\left|\sum_{j=n+1}^{k} \Delta u_{j}\right|=0 .
$$

It is clear from Definition 3 that slow oscillation of $\left(u_{n}\right)$ implies $\Delta u_{n}=o(1)$.

By Littlewood's condition $n \Delta u_{n}=O(1)$ [8] and slow oscillation of $\left(u_{n}\right)$, Dik et al. [6] obtained some Tauberian-like theorems to recover subsequential convergence of $\left(u_{n}\right)$ from its boundedness. The condition $n \Delta u_{n}=O\left(A_{n}\right)$, where $\left(A_{n}\right)$ is an unbounded sequence, is used as a Tauberian-like condition for the recovery of subsequential convergence of $\left(u_{n}\right)$ from its boundedness by Dik et al. [6]. Later in [1], Çanak and Dik introduced some Tauberian-like conditions $\omega_{n, 1}^{(m)}(u)=O\left(A_{n}\right)$, where $\left(A_{n}\right)$ is an unbounded sequence, and slow oscillation of $\left(\Delta \sigma_{n, 1}\left(\omega^{(m)}(u)\right)\right)$ for recovering subsequential convergence of the sequence of $\left(u_{n}\right)$ from its boundedness.

In this paper we prove that subsequential convergence of $\left(u_{n}\right)$ follows from its boundedness under the condition given in terms of the weighted general control modulo of the oscillatory behavior of integer order of the sequence $\left(u_{n}\right)$.

\section{MAIN RESULTS}

The main theorem of this paper involves the concepts of a regularly varying sequence of index $\alpha>-1$ and slowly oscillating sequence.

Definition 4 ([7]). A positive sequence $(R(n))$ is said to be regularly varying of index $\alpha>-1$ if

$$
\lim _{n \rightarrow \infty} \frac{R([\lambda n])}{R(n)}=\lambda^{\alpha}, \lambda>0, \alpha>-1 .
$$

The following theorems generalize some theorems in [1] that are exactly given in terms of the weighted general control modulo of the oscillatory behavior of the sequence $\left(u_{n}\right)$.

Theorem 2. Let $\left(u_{n}\right)$ be a bounded sequence and

$$
\frac{P_{n-1}}{p_{n}}=O(n)
$$

Let $\left(A_{n}\right)$ be a sequence such that

$$
\frac{1}{P_{n}} \sum_{k=0}^{n} p_{k}\left|A_{k}\right|^{\mathfrak{p}}=O(1), \mathfrak{p}>1
$$


for some regularly varying sequence $\left(P_{n}\right)$ of index $\alpha>-1$. If

$$
\omega_{n, p}^{(m)}(u)=O\left(A_{n}\right)
$$

then $\left(u_{n}\right)$ is subsequentially convergent.

Theorem 3. Let $\left(u_{n}\right)$ be a bounded sequence and let $\left(P_{n}\right)$ be regularly varying of index $\alpha>-1$. If $\left(\Delta \sigma_{n, p}^{(1)}\left(\omega^{(m)}(u)\right)\right)$ is slowly oscillating for some nonnegative integer $m$, then $\left(u_{n}\right)$ is subsequentially convergent.

\section{IDENTITIES AND A LEMMA}

In this section, we present some identities and a lemma to be used in the proof of the main theorems.

The identities

$$
\frac{P_{n-1}}{p_{n}} \Delta \sigma_{n, p}^{(m)}(u)=V_{n, p}^{(m-1)}(\Delta u)
$$

and

$$
\sigma_{n, p}^{(1)}\left(\frac{P_{n-1}}{p_{n}} \Delta V_{n, p}^{(0)}(\Delta u)\right)=\frac{P_{n-1}}{p_{n}} \Delta V_{n, p}^{(1)}(\Delta u) .
$$

are proved by Totur and Çanak [10].

For a sequence $u=\left(u_{n}\right)$, we define

$$
\left(\frac{P_{n-1}}{p_{n}} \Delta\right)_{m} u_{n}=\left(\frac{P_{n-1}}{p_{n}} \Delta\right)_{m-1}\left(\frac{P_{n-1}}{p_{n}} \Delta u_{n}\right)=\frac{P_{n-1}}{p_{n}} \Delta\left(\left(\frac{P_{n-1}}{p_{n}} \Delta\right)_{m-1} u_{n}\right) \text {, }
$$

where $\left(\frac{P_{n-1}}{p_{n}} \Delta\right)_{0} u_{n}=u_{n}$, and $\left(\frac{P_{n-1}}{p_{n}} \Delta\right)_{1} u_{n}=\frac{P_{n-1}}{p_{n}} \Delta u_{n}$.

A different representation of the weighted general control modulo of integer order $m \geq 1$ of a sequence $\left(u_{n}\right)$ is given by the identity

$$
\omega_{n, p}^{(m)}(u)=\left(\frac{P_{n-1}}{p_{n}} \Delta\right)_{m} V_{n, p}^{(m-1)}(\Delta u)
$$

in ([10]).

We note that any $\left(P_{n}\right)$ with $\lim _{n \rightarrow \infty} \frac{P_{n}}{n}=1$ is regularly varying of index 1 .

Lemma 1. Let $\left(P_{n}\right)$ be regularly varying of index $\alpha>-1$. If $\left(u_{n}\right)$ is limitable to $s$ by the weighted mean method $\left(\bar{N}, p_{n}\right)$ and slowly oscillating, then $\left(u_{n}\right)$ converges to $s$.

Proof. Assume that $\left(P_{n}\right)$ be regularly varying of index $\alpha>-1$. If $\left(u_{n}\right)$ is slowly oscillating, then $\left(V_{n, p}^{(0)}(\Delta u)\right)$ is slowly oscillating (see [3]). Since $u_{n} \rightarrow s\left(\bar{N}, p_{n}\right)$ and $\left(V_{n, p}^{(0)}(\Delta u)\right)$ is slowly oscillating, the proof is completed by [2, Theorem 6]. 


\section{Proofs}

In this section, proofs of theorems and corollaries are given.

Proof of Theorem 2. By (2.3), it follows that the sequence $\left(\sum_{j=1}^{n} \frac{p_{j} A_{j}}{P_{j-1}}\right)$ is slowly oscillating. Indeed,

$$
\begin{aligned}
\max _{n+1 \leq k \leq[\lambda n]}\left|\sum_{j=n+1}^{k} \frac{p_{j} A_{j}}{P_{j-1}}\right| & \leq \max _{n+1 \leq k \leq[\lambda n]} \sum_{j=n+1}^{k}\left|\frac{p_{j} A_{j}}{P_{j-1}}\right| \\
& \leq \sum_{j=n+1}^{[\lambda n]} \frac{p_{j}\left|A_{j}\right|}{P_{j-1}} \leq \frac{1}{P_{n}} \sum_{j=n+1}^{[\lambda n]} p_{j}\left|A_{j}\right| \\
& =\frac{P_{[\lambda n]}-P_{n}}{P_{n}} \frac{1}{P_{[\lambda n]}-P_{n}} \sum_{j=n+1}^{[\lambda n]} p_{j}\left|A_{j}\right| \\
& \leq \frac{P_{[\lambda n]}-P_{n}}{P_{n}} \frac{1}{\left(P_{[\lambda n]}-P_{n}\right)^{\frac{1}{\mathfrak{p}}}}\left(\sum_{j=n+1}^{[\lambda n]} p_{j}\left|A_{j}\right|^{\mathfrak{p}}\right)^{\frac{1}{\mathfrak{p}}} \\
& =\frac{\left(P_{[\lambda n]}-P_{n}\right)^{1-\frac{1}{\mathfrak{p}}}}{P_{n}}\left(\sum_{j=n+1}^{[\lambda n]} p_{j}\left|A_{j}\right|^{\mathfrak{p}}\right)^{\frac{1}{\mathfrak{p}}} \\
& =\frac{\left(P_{[\lambda n]}-P_{n}\right)^{\frac{1}{\mathfrak{q}}}}{P_{n}}\left(\sum_{j=n+1}^{[\lambda n]} p_{j}\left|A_{j}\right|^{\mathfrak{p}}\right)^{\frac{1}{\mathfrak{p}}}, \\
& =\frac{\left(P_{[\lambda n]}-P_{n}\right)^{\frac{1}{\mathfrak{q}}}}{\left(P_{n}\right)^{\frac{1}{\mathfrak{q}}}}\left(\frac{P_{[\lambda n]}}{P_{n}} \frac{1}{P_{[\lambda n]}} \sum_{j=0}^{[\lambda n]} p_{j}\left|A_{j}\right|^{\mathfrak{p}}\right)^{\frac{1}{\mathfrak{p}}} \\
& =\frac{\left(P_{[\lambda n]}-P_{n}\right)^{\frac{1}{\mathfrak{q}}}}{\left(P_{n}\right)^{\frac{1}{\mathfrak{q}}}} \frac{\left(P_{[n n]}\right)^{\frac{1}{\mathfrak{p}}}}{\left(P_{n}\right)^{\frac{1}{\mathfrak{p}}}}\left(\frac{1}{P_{[\lambda n]}} \sum_{j=0}^{[\lambda n]} p_{j}\left|A_{j}\right|^{\mathfrak{p}}\right)^{\frac{1}{\mathfrak{p}}},
\end{aligned}
$$

where $\frac{1}{\mathfrak{p}}+\frac{1}{\mathfrak{q}}=1$. Taking limsup of both sides as $n \rightarrow \infty$, we get

$$
\limsup _{n} \max _{n+1 \leq k \leq[\lambda n]}\left|\sum_{j=n+1}^{k} \frac{p_{j} A_{j}}{P_{j-1}}\right|
$$




$$
\begin{aligned}
& \leq \limsup _{n} \frac{\left(P_{[\lambda n]}-P_{n}\right)^{\frac{1}{\mathfrak{q}}}}{\left(P_{n}\right)^{\frac{1}{\mathfrak{q}}}} \frac{\left(P_{[\lambda n]}\right]^{\frac{1}{\mathfrak{p}}}}{\left(P_{n}\right)^{\frac{1}{\mathfrak{p}}}} \limsup _{n}\left(\frac{1}{P_{[\lambda n]}} \sum_{j=0}^{[\lambda n]} p_{j}\left|A_{j}\right|^{\mathfrak{p}}\right)^{\frac{1}{\mathfrak{p}}} \\
& =\lim _{n}\left(\frac{P_{[\lambda n]}-P_{n}}{P_{n}}\right)^{\frac{1}{\mathfrak{q}}} \lim _{n}\left(\frac{P_{[\lambda n]}}{P_{n}}\right)^{\frac{1}{\mathfrak{p}}} \limsup _{n}\left(\frac{1}{P_{[\lambda n]}} \sum_{j=0}^{[\lambda n]} p_{j}\left|A_{j}\right|^{\mathfrak{p}}\right)^{\frac{1}{\mathfrak{p}}}
\end{aligned}
$$

Since $\left(P_{n}\right)$ is regularly varying of index $\alpha>-1$, we have

$$
\limsup _{n} \max _{n+1 \leq k \leq[\lambda n]}\left|\sum_{j=n+1}^{k} \frac{p_{j} A_{j}}{P_{j-1}}\right| \leq C\left(\lambda^{\alpha}-1\right)^{\frac{1}{\mathfrak{q}}}\left(\lambda^{\alpha}\right)^{\frac{1}{\mathfrak{p}}} .
$$

for some $C>0$.

Finally, taking limsup of both sides as $\lambda \rightarrow 1+$, we obtain

$$
\lim _{\lambda \rightarrow 1^{+}} \limsup _{n} \max _{n+1 \leq k \leq[\lambda n]}\left|\sum_{j=n+1}^{k} \frac{p_{j} A_{j}}{P_{j-1}}\right| \leq C \lim _{\lambda \rightarrow 1^{+}}\left(\lambda^{\alpha}-1\right)^{\frac{1}{\mathfrak{q}}}\left(\lambda^{\alpha}\right)^{\frac{1}{\mathfrak{p}}}=0 .
$$

Slow oscillation of $\left(\sum_{j=1}^{n} \frac{p_{j} A_{j}}{P_{j-1}}\right)$ implies that $\frac{p_{j} A_{j}}{P_{j-1}}=o(1)$. Since $\omega_{n, p}^{(m)}(u)=$ $O\left(A_{n}\right)$, it follows from

$$
\omega_{n, p}^{(m)}(u)=\frac{P_{n-1}}{p_{n}} \Delta\left(\left(\frac{P_{n-1}}{p_{n}} \Delta\right)_{m-1} V_{n, p}^{(m-1)}(\Delta u)\right)=O\left(A_{n}\right)
$$

that

$$
\Delta \sigma_{n, p}^{(1)}\left(\omega^{(m-1)}(u)\right)=\Delta\left(\left(\frac{P_{n-1}}{p_{n}} \Delta\right)_{m-1} V_{n, p}^{(m-1)}(\Delta u)\right)=o(1) .
$$

Since $\left(u_{n}\right)$ is bounded, $\left(\sigma_{n, p}^{(1)}\left(\omega^{(m-2)}(u)\right)\right)$ is bounded for every nonnegative integer $m$. From the identity

$$
\begin{aligned}
\Delta\left(\left(\frac{P_{n-1}}{p_{n}} \Delta\right)_{m-2} V_{n, p}^{(m-2)}(\Delta u)\right)= & \frac{p_{n}\left(\frac{P_{n-1}}{p_{n}} \Delta\right)_{m-2} V_{n, p}^{(m-2)}(\Delta u)}{P_{n-1}} \\
& +\Delta\left(\left(\frac{P_{n-1}}{p_{n}} \Delta\right)_{m-1} V_{n, p}^{(m-1)}(\Delta u)\right),
\end{aligned}
$$

it follows that

$$
\sigma_{n, p}^{(1)}\left(\omega^{(m-2)}(u)\right)=\Delta\left(\left(\frac{P_{n-1}}{p_{n}} \Delta\right)_{m-2} V_{n, p}^{(m-2)}(\Delta u)\right)=o(1), n \rightarrow \infty .
$$


Continuing in this manner, we obtain that

$$
\Delta \sigma_{n, p}^{(1)}\left(\omega^{(0)}(u)\right)=\Delta V_{n, p}^{(0)}(\Delta u)=o(1) .
$$

Using (1.1), we have

$$
\Delta u_{n}=\frac{p_{n} V_{n, p}^{(0)}(\Delta u)}{P_{n-1}}+\Delta V_{n}^{(0)}(\Delta u)=o(1) .
$$

By Theorem 1 we have the proof.

Corollary 1. Let $\left(u_{n}\right)$ be a bounded sequence and $\left(A_{n}\right)$ be a sequence such that

$$
\frac{1}{n} \sum_{k=1}^{n}\left|A_{k}\right|^{\mathfrak{p}}=O(1), \mathfrak{p}>1
$$

If $\omega_{n}^{(m)}=O\left(A_{n}\right)$, then $\left(u_{n}\right)$ is subsequentially convergent.

Proof. Take $p_{n}=1$ for every nonnegative integer $n$ in Theorem 2 .

Corollary 1 was given by Çanak and Dik [1].

Proof of Theorem 3. Since $\left(u_{n}\right)$ is bounded, then $\left(\sigma_{n, p}^{(1)}\left(\omega^{(m)}(u)\right)\right)$ is bounded for every nonnegative integer $m$. Thus, we get

$$
\sigma_{n, p}^{(1)}\left(\Delta \sigma^{(1)}\left(\omega^{(m)}(u)\right)\right)=\frac{p_{n}(n \Delta)_{m} V_{n, p}^{(m)}(\Delta u)}{P_{n-1}}=o(1) .
$$

Since $\left(\Delta \sigma_{n, p}^{(1)}\left(\omega^{(m)}(u)\right)\right)$ is slowly oscillating, applying Lemma 1 to the sequence $\left(\Delta \sigma_{n, p}^{(1)}\left(\omega^{(m)}(u)\right)\right)$ we obtain that

$$
\Delta \sigma_{n, p}^{(1)}\left(\omega^{(m)}(u)\right)=\Delta\left(\left(\frac{P_{n-1}}{p_{n}} \Delta\right)_{m} V_{n, p}^{(m)}(\Delta u)\right)=o(1) .
$$

Since $\left(\sigma_{n, p}^{(1)}\left(\omega^{(m-1)}(u)\right)\right)$ is bounded for every nonnegative integer $m$, by the identity

$$
\begin{aligned}
\Delta\left(\left(\frac{P_{n-1}}{p_{n}} \Delta\right)_{m-1} V_{n, p}^{(m-1)}(\Delta u)\right)= & \frac{p_{n}\left(\frac{P_{n-1}}{p_{n}} \Delta\right)_{m} V_{n, p}^{(m)}(\Delta u)}{P_{n-1}} \\
& +\Delta\left(\left(\frac{P_{n-1}}{p_{n}} \Delta\right)_{m} V_{n, p}^{(m)}(\Delta u)\right),
\end{aligned}
$$

it follows that

$$
\Delta \sigma_{n, p}^{(1)}\left(\omega^{(m-1)}(u)\right)=\Delta\left(\left(\frac{P_{n-1}}{p_{n}} \Delta\right)_{m-1} V_{n, p}^{(m-1)}(\Delta u)\right)=o(1) .
$$

The rest of the proof is as in the proof of Theorem 2.

Corollary 2. Let $\left(u_{n}\right)$ be a bounded sequence. If $\left(\sigma_{n, p}^{(1)}\left(\omega^{(m)}(u)\right)\right)$ is slowly oscillating for some nonnegative integer $m$, then $\left(u_{n}\right)$ is subsequentially convergent. 
Proof. Proof follows from the fact that $\lim _{n} \Delta u_{n}=0$ for every slowly oscillating sequence $\left(u_{n}\right)$.

Corollary 3. Let $\left(u_{n}\right)$ be a bounded sequence. If $\left(\Delta \sigma_{n}^{(1)}\left(\omega^{(m)}(u)\right)\right)$ is slowly oscillating for some nonnegative integer $m$, then $\left(u_{n}\right)$ is subsequentially convergent.

Proof. Take $p_{n}=1$ for every nonnegative integer $n$ in Theorem 3 .

Corollary 3 was given by Çanak and Dik [1].

\section{REFERENCES}

[1] Çanak, İ. and Dik, M., "Some conditions under which subsequential convergence follows from boundedness," Appl. Math. Lett., vol. 21, no. 9, pp. 957-960, 2008.

[2] Çanak, İ and Totur, Ü., "Some Tauberian theorems for the weighted mean methods of summability," Comput. Math. Appl., vol. 62, no. 6, pp. 2609-2615, 2011.

[3] Çanak, İ. and Totur, Ü., "Tauberian theorems for the $(J, p)$ summability method," Appl. Math. Lett., vol. 25, no. 10, pp. 1430-1434, 2012.

[4] Çanak, İ and Totur, Ü. , "An extended Tauberian theorem for the weighted mean method of summability," Ukrainian Math. J., vol. 65, no. 7, pp. 929-936, 2013.

[5] Dik, F., "Tauberian theorems for convergence and subsequential convergence with moderately oscillatory behavior," Math. Morav., vol. 5, pp. 19-56, 2001.

[6] Dik, F., Dik, M. and Çanak, İ., "Applications of subsequential Tauberian theory to classical Tauberian theory," Appl. Math. Lett., vol. 20, no. 8, pp. 946-950, 2007.

[7] Karamata, J., "Sur certains "Tauberian theorems" de M. M. Hardy et Littlewood," Mathematica, vol. 3, pp. 33-48, 1930.

[8] Littlewood, J. E., "The converse of Abel's theorem on power series," Proc. London. Math. Soc., vol. 9, pp. 434-448, 1911, doi: 10.1112/plms/s2-9.1.434.

[9] Stanojević, Č. V., Analysis of Divergence: Control and Management of Divergent Process, ser. Graduate Research Seminar Lecture Notes, 1998.

[10] Totur, Ü. and Çanak, İ., "Some general Tauberian conditions for the weighted mean method of summability," Comput. Math. Appl., vol. 63, no. 5, pp. 999-1006, 2012, doi: 10.1016/j.camwa.2011.12.005.

Authors' addresses

İbrahim Çanak

Ege University, Department of Mathematics, 35100 Izmir, Turkey

E-mail address: ibrahim.canak@ege.edu.tr

Ümit Totur

Adnan Menderes University, Department of Mathematics, 09010 Aydin, Turkey

E-mail address: utotureadu.edu.tr 